\title{
Canonical analysis of new non-relativistic string action and uniform light-cone gauge formulation
}

\section{J. Klusoň}

Department of Theoretical Physics and Astrophysics, Faculty of Science, Masaryk University, Kotlářská 2, 611 37, Brno, Czech Republic

E-mail: klu@physics.muni.cz

ABSTRACT: We perform canonical analysis of new non-relativistic string action that was found recently in [32]. We also discuss its gauge fixed form.

KEYwords: Bosonic Strings, String Duality

ArXiv EPrint: 2108.13804 


\section{Contents}

1 Introduction and summary 1

2 New non-relativistic string $\quad 2$

3 Hamiltonian formalism 4

4 Uniform light-cone gauge $\quad 6$

\section{Introduction and summary}

There are several examples of consistent two dimensional field theories defined on string world-sheet. One such an example is non-relativistic string theory that was originally introduced twenty years ago in $[1,2]$. Renewed interest in this theory began with remarkable paper [3] where new stringy Newton-Cartan geometry was introduced by specific limiting procedure with foliation of space-time into two dimensional longitudinal subspace and transverse one where longitudinal subspace has origin in the idea that the non-relativistic space-time is probed by two dimensional object-string. This proposal was subsequently studied in many papers, see for example [5-20,32]. Another, seemeangly different approach for definition of non-relativistic string theory, was presented in remarkable paper [4] where non-relativistic string in torsional Newton-Cartan background was defined by null dimensional reduction from higher dimensional space-time with light-like isometry, for further works see for example [21-31]. Finally the relation between these two seamingly different non-relativistic string theories was found in [13].

Recently new form of non-relativistic string theory was formulated in [32]. This new Newton-Cartan geometry is called as torsional string Newton-Cartan (TSNC) geometry. In this formulation we can see close analogy between non-relativistic limit of point particle and its coupling to Newton-Cartan geometry. Explicitly, in the point particle case the particle naturally couples to mass form $m_{\mu}$ while in case of non-relativistic string in (TSNC) geometry the corresponding object is two form gauge field $m_{\mu \nu}$. This idea was further elaborated in [32] where new non-relativistic string algebra, called as F-string Galilei algebra, was found. The non-relativistic string action in TSNC geometry was studied very carefully in [32] and brings new opportunities for the analysis of non-relativistic theories.

The goal of this paper is to find canonical formulation of this theory and further study its gauge fixed version that could be potentially useful for non-relativistic expansion of various string theory backgrounds, as for example $A d S_{5} \times S^{5}$. As in case of ordinary string we find that the bare Hamiltonian vanishes which is a consequence of the fact that nonrelativistic string is diffeomorphism invariant theory on two dimensional world-sheet. As a result we have to find two primary constraints. It is rather easy to determine spatial 
diffeomorphism constraint starting from the definition of canonical momenta. The situation is more difficult in case of Hamiltonian constraint however it is still simpler than in case of non-relativistic string in stringy Newton-Cartan background [19]. This is again very remarkable property of the new formulation of non-relativistic string. We also show that these two constraints are first class constraints that prove consistency of this theory.

As the next step we use this Hamiltonian formalism to formulate non-relativistic theory in the uniform light-cone gauge. Such a specific gauge was introduced in the context of study of string theory on $A d S_{5} \times S^{5}$ background [33, 35-37]. ${ }^{1}$ We show that it is possible to impose this gauge in case of non-relativistic string as well. Then we are able to find Hamiltonian on the reduced phase space. This Hamiltonian could be useful for the study of non-relativistic limit of some backgrounds as for example non-relativistic limit of $A d S_{5} \times S^{5}$. Similar problem was recently studied in remarkable paper [23]. It would be certainly nice to perform similar analysis in case of new non-relativistic string action [32]. We hope to return to this problem in future.

The structure of this paper is as follows. In the next section we review construction of new non-relativistic string action as was performed recently in [32]. Then in section (3) we find Hamiltonian form of this theory. Finally in section (4) we find gauge fixed form of this theory switching to uniform light-cone gauge.

\section{New non-relativistic string}

In this section we review construction of recently proposed non-relativistic string action [32]

$$
S=-\frac{T}{2} \int d^{2} \sigma \sqrt{-\tau}\left[\tau^{\alpha \beta} h_{M N}+\epsilon^{\alpha \beta} m_{M N}\right] \partial_{\alpha} x^{M} \partial_{\beta} x^{N} .
$$

Our goal is to show how such an action can be derived, following [32]. Let us introduce vielbein $e_{\frac{a}{M}}$ so that the target space metric has the form

$$
g_{M N}=e_{\frac{a}{M}} e^{\frac{b}{N}} \eta_{\underline{a b}},
$$

where we use the similar notation as in [32] so that frame indices are $\underline{a}, \underline{b}=0, \ldots, D-1$ and where $\eta_{\underline{a b}}=\operatorname{diag}(-1,1, \ldots, 1)$. Note that space-time indices are $M, N=0,1, \ldots, D-1$. Following [32] we also introduce parametrization of NSNS two form $B_{M N}$ as

$$
B_{M N}=\frac{1}{2} \eta_{\underline{a b}}\left(e^{\frac{a}{M}} \pi_{\frac{b}{N}}-e_{\frac{a}{N}} \pi_{\frac{b}{M}}\right) .
$$

To begin with let us write Nambu-Goto form of the action for relativistic string in general background

$$
S=-c T_{F} \int d^{2} \sigma \sqrt{-\operatorname{det} g_{\alpha \beta}}-c T_{F} \int d^{2} \sigma \frac{1}{2} \epsilon^{\alpha \beta} B_{\alpha \beta},
$$

where $\epsilon^{01}=1=-\epsilon_{01}$ and $T_{F}$ is string tension. Note that $M, N=0,1, \ldots, D-1$. As in [32] we introduce indices $\underline{a}=(A, a)$ corresponding to directions longitudinal and transverse to

\footnotetext{
${ }^{1}$ For review, see for example [34].
} 
string world-sheet where $A=0,1$ are longitudinal and $a=2, \ldots, D-1$ are transverse. Then we have

$$
e_{M}^{\frac{a}{M}}=\left(c E_{M}^{A}, e_{M}^{a}\right), \quad \pi_{M}^{a}=\left(c \Pi_{M}^{A}, \pi_{M}^{a}\right)
$$

so that

$$
\begin{aligned}
g_{\alpha \beta} & =c^{2} \eta_{A B} E_{\alpha}{ }^{A} E_{\beta}{ }^{B}+\delta_{a b} e_{\alpha}^{a} e_{\beta}^{b}, \\
B_{\alpha \beta} & =\frac{1}{2} c^{2} \eta_{A B}\left(E_{\alpha}{ }^{A} \Pi_{\beta}{ }^{B}-E_{\beta}{ }^{A} \Pi_{\alpha}{ }^{B}\right)+\frac{1}{2} \delta_{a b}\left(e_{\alpha}^{a} \pi_{\beta}^{b}-e_{\beta}{ }^{a} \pi_{\alpha}^{b}\right) .
\end{aligned}
$$

Then as in [32] we parametrize longitudinal components as

$$
E_{M}^{A}=\tau_{M}^{A}+\frac{1}{2 c^{2}} \pi_{M}^{B} \epsilon_{B}^{A}, \quad \Pi_{M}^{A}=\epsilon_{B}^{A} \tau_{M}^{B}+\frac{1}{2 c^{2}} \pi_{M}^{A},
$$

where $\epsilon_{B}^{A}=\epsilon_{B C} \eta^{C A}$. Inserting (2.7) into (2.2) we finally get

$$
\begin{aligned}
g_{\alpha \beta}= & c^{2} \tau_{\alpha \beta}+\frac{1}{2} \eta_{A B}\left(\tau_{\alpha}{ }^{A} \pi_{\beta}{ }^{C} \epsilon_{C}^{B}+\tau_{\beta}{ }^{B} \pi_{\alpha}{ }^{C} \epsilon_{C}^{B}\right)+h_{\alpha \beta} \\
& +\frac{1}{4 c^{2}} \eta_{A B} \pi_{\alpha}^{C} \epsilon_{C}{ }^{A} \pi_{\beta}{ }^{D} \epsilon_{D}^{B},
\end{aligned}
$$

where

$$
\tau_{\alpha \beta}=\tau_{\alpha}{ }^{A} \tau_{\beta}{ }^{B} \eta_{A B}, \quad h_{\alpha \beta}=e_{\alpha}^{a} e_{\beta}^{b} \delta_{a b} .
$$

Then it is easy to see that

$$
\sqrt{-\operatorname{det} g_{\alpha \beta}}=c^{2} \sqrt{-\operatorname{det} \tau_{\alpha \beta}}\left(1+\frac{1}{2 c^{2}} \tau^{\alpha \beta} h_{\beta \alpha}\right)+\frac{1}{2} \epsilon^{\alpha \beta} \tau_{\beta}{ }^{A} \eta_{A B} \pi_{\alpha}{ }^{B},
$$

where $\tau^{\alpha \beta}=\tau_{A}^{\alpha} \tau^{\beta}{ }_{B} \eta^{A B}$ is $2 \times 2$ matrix inverse to $\tau_{\alpha \beta}$. We also introduced $2 \times 2$ twobein $\tau_{A}^{\alpha}$ that obeys the condition

$$
\tau_{\alpha}^{A} \tau_{A}^{\beta}=\delta_{\alpha}^{\beta}, \quad \tau_{\alpha}^{A} \tau_{B}^{\alpha}=\delta_{B}^{A}
$$

Explicitly we have

$$
\tau_{A}^{\alpha}=\frac{1}{\operatorname{det} \tau_{\alpha}{ }^{A}}\left(\begin{array}{cc}
\tau_{1}^{1} & -\tau_{0}{ }^{1} \\
-\tau_{1}{ }^{0} & \tau_{0}{ }^{0}
\end{array}\right)=-\frac{1}{\sqrt{-\operatorname{det} \tau_{\alpha \beta}}} \epsilon^{\alpha \beta} \epsilon_{A B} \tau_{\beta}{ }^{B}
$$

where $\epsilon_{01}=-1=-\epsilon_{10}$. Further, using (2.6) we obtain

$$
\frac{1}{2} \epsilon^{\alpha \beta} B_{\alpha \beta}=-c^{2} \sqrt{-\operatorname{det} \tau}+\frac{1}{2} \epsilon^{\alpha \beta} \mathbf{B}_{\alpha \beta},
$$

where

$$
\mathbf{B}_{\alpha \beta}=\frac{1}{2} \delta_{a b}\left(e_{\alpha}^{a} \pi_{\beta}^{b}-e_{\beta}^{a} \pi_{\alpha}^{b}\right) .
$$

We further have

$$
\tau_{A}^{M} h_{M N}=0, \quad \tau_{A}^{M} \tau_{B}^{N} \mathbf{B}_{M N}=0 .
$$


We see that two terms proportional to determinant of $\tau$ cancel each other. As a result we obtain non-relativistic action in the form

$$
S=-\frac{T}{2} \int d^{2} \sigma\left[\sqrt{-\tau} \tau^{\alpha \beta} h_{\alpha \beta}+\epsilon^{\alpha \beta} m_{\alpha \beta}\right]
$$

where we introduced rescaled tension $c T_{F}=T$ and we have taken the limit $c \rightarrow \infty$. Finally we also defined $m_{M N}$ as

$$
m_{M N}=\frac{1}{2} \eta_{A B}\left[\tau_{M}^{A} \pi_{N}^{B}-\tau_{N}^{A} \pi_{M}^{B}\right]+\frac{1}{2} \delta_{a b}\left[e_{M}^{a} \pi_{N}^{b}-e_{N}^{a} \pi_{M}^{b}\right],
$$

where $m_{\alpha \beta}=m_{M N} \partial_{\alpha} x^{M} \partial_{\beta} x^{N}$ is pull back of $m_{M N}$ to the world-sheet of the string. Before we proceed further it is instructive to stress following point. Using the fact that

$$
-\frac{1}{2} \sqrt{-\operatorname{det} \tau} \tau^{\alpha \beta}=\frac{1}{2} \epsilon^{\alpha \beta}\left(\tau_{\alpha}{ }^{A} \eta_{A B} \pi_{\beta}^{B}-\tau_{\beta}{ }^{A} \eta_{A B} \pi_{\alpha}{ }^{B}\right)
$$

it is easy to see that the action (2.16) can be rewritten into the form

$$
S=-\frac{T}{2} \int d^{2} \sigma\left[\sqrt{-\tau} \tau^{\alpha \beta} \bar{h}_{\alpha \beta}+\epsilon^{\alpha \beta} \mathbf{B}_{\alpha \beta}\right]
$$

where

$$
\bar{h}_{\alpha \beta}=\bar{h}_{M N} \partial_{\alpha} x^{M} \partial_{\beta} x^{N}, \quad \bar{h}_{M N}=h_{M N}-\frac{1}{2} \epsilon_{A B}\left(\tau_{M}^{A} \pi_{N}^{B}+\tau_{N}^{A} \pi_{M}^{B}\right) .
$$

If we identify $m_{M}^{A}=-\frac{1}{2} \eta^{A B} \epsilon_{B C} \pi_{M}^{C}$ we obtain standard form of non-relativistic action with an important difference that $\mathbf{B}$ is not arbitrary NSNS two form but it is explicitly defined in (2.14).

In this section we gave brief description of procedure that was used in [32] in order do find non-relativistic action (2.16). In the next section we proceed to the canonical analysis of this action.

\section{Hamiltonian formalism}

In order to find canonical form of the action (2.16) it is useful to rewrite it into the form

$$
S=-\frac{T}{2} \int d^{2} \sigma\left[\operatorname{det} \tau_{\gamma}^{C} \tau_{A}^{\alpha} \tau_{B}^{\beta} \eta^{A B} h_{\alpha \beta}+\epsilon^{\alpha \beta} m_{\alpha \beta}\right],
$$

where we used the fact that $\sqrt{-\operatorname{det} \tau}=\operatorname{det} \tau_{\gamma}{ }^{A}$. From (3.1) we determine conjugate momenta

$$
\begin{aligned}
p_{M}=\frac{\partial \mathcal{L}}{\partial\left(\partial_{0} x^{M}\right)}= & -\frac{T}{2} \tau_{M}^{A} \tau_{A}^{0}{ }_{A} \operatorname{det} \tau_{\gamma}{ }^{C} \tau^{\alpha \beta} h_{\alpha \beta}+T \operatorname{det} \tau_{\gamma}{ }^{C} \tau^{0}{ }_{A} \eta^{A B} \tau^{\beta}{ }_{B} \tau_{M}^{D} \tau_{D}^{\alpha} h_{\alpha \beta} \\
& -T \operatorname{det} \tau_{\alpha}{ }^{D} \tau^{0 \alpha} h_{\alpha M}-T m_{M N} \partial_{1} x^{N}
\end{aligned}
$$

and where we used the fact that

$$
\begin{aligned}
\frac{\partial \operatorname{det} \tau_{\gamma}{ }^{C}}{\partial\left(\partial_{0} x^{M}\right)} & =\frac{\partial\left(\tau_{\alpha}{ }^{A}\right)}{\partial\left(\partial_{0} x^{M}\right)} \tau_{A}^{\alpha} \operatorname{det} \tau_{\gamma}{ }^{C}=\tau_{M}^{A} \tau^{0}{ }_{A} \operatorname{det} \tau_{\gamma}{ }^{C} \\
\frac{\partial \tau^{\alpha \beta}}{\partial\left(\partial_{0} x^{M}\right)} h_{\alpha \beta} & =2 \frac{\partial\left(\tau_{A}^{\alpha}\right)}{\partial\left(\partial_{0} x^{M}\right)} \tau_{B}^{\beta} \eta^{A B} h_{\alpha \beta}=-2 \tau^{0}{ }_{A} \eta^{A B} \tau_{B}^{\beta}{ }_{B} \tau_{M}{ }^{C} \tau_{C}^{\alpha} h_{\alpha \beta}
\end{aligned}
$$


using

$$
\frac{\partial\left(\tau_{A}^{\alpha}\right)}{\partial\left(\partial_{0} x^{M}\right)}=-\tau_{A}^{\gamma} \frac{\partial \tau_{\gamma}^{B}}{\partial\left(\partial_{0} x^{M}\right)} \tau_{B}^{\alpha}=-\tau_{A}^{0} \tau_{M}^{B} \tau_{B}^{\alpha}
$$

Now with the help of the definition of the momenta $p_{M}$ given in (3.2) we determine bare Hamiltonian

$$
H_{B}=\int d \sigma\left(p_{M} \partial_{0} x^{M}-\mathcal{L}\right)=0
$$

as expected. On the other hand from definition of momenta $p_{M}$ given in (3.2) we obtain

$$
\partial_{1} x^{M} p_{M}=-\frac{T}{2} \tau_{1}{ }^{A} \tau_{A}^{0} \operatorname{det} \tau_{\gamma}{ }^{D} \tau^{\alpha \beta} h_{\beta \alpha}+T \operatorname{det} \tau_{\gamma}{ }^{D} \tau^{0 \beta} \tau_{1}{ }^{C} \tau_{C}^{\alpha} h_{\alpha \beta}-T \operatorname{det} \tau_{\gamma}{ }^{D} \tau^{0 \alpha} h_{\alpha 1}=0
$$

and hence we have following constraint

$$
\mathcal{H}_{\sigma}=p_{M} \partial_{\sigma} x^{M} \approx 0 .
$$

To proceed further we introduce $\Pi_{M}$ as

$$
\Pi_{M}=p_{M}+T m_{M N} \partial_{1} x^{N} .
$$

Then from (3.2) we obtain

$$
\begin{aligned}
\Pi_{M} \tau_{A}^{M} \eta^{A B} \epsilon_{B D} \tau_{1}{ }^{D}= & -\frac{T}{2} \tau^{0}{ }_{A} \eta^{A B} \epsilon_{B C} \tau_{1}{ }^{C} \operatorname{det} \tau_{\gamma}{ }^{D} \tau^{\alpha \beta} h_{\beta \alpha}+ \\
& +T \operatorname{det} \tau_{\gamma}{ }^{D} \tau^{0 \beta} h_{\beta \alpha} \tau_{A}^{\alpha} \eta^{A B} \epsilon_{B D} \tau_{1}{ }^{D}=\frac{T}{2}\left(\operatorname{det} \tau_{\gamma}{ }^{D}\right)^{2} \tau^{0 \alpha} h_{\alpha \beta} \tau^{\beta 0}+\frac{T}{2} h_{11} .
\end{aligned}
$$

Further, using (3.2) we also find that $\Pi_{M} h^{M N} \Pi_{N}$ is equal to

$$
\Pi_{M} h^{M N} \Pi_{N}=T^{2}\left(\operatorname{det} \tau_{\gamma}{ }^{D}\right)^{2} \tau^{0 \alpha} \partial_{\alpha} x^{M} h_{M N} \partial_{\beta} x^{N} \tau^{\beta 0} .
$$

Collecting these terms together we obtain following Hamiltonian constraint

$$
-2 T \Pi_{M} \tau_{A}^{M} \eta^{A B} \epsilon_{B D} \tau_{1}^{D}+T^{2} h_{11}+\Pi_{M} h^{M N} \Pi_{N} \equiv \mathcal{H}_{\tau} \approx 0 .
$$

In summary, we have theory with two primary constraints $\mathcal{H}_{\tau} \approx 0, \mathcal{H}_{\sigma} \approx 0$. It is remarkable that the Hamiltonian constraint (3.11) has simpler form than in case of the Hamiltonian constraint that was found in case of non-relativistic string in stringy Newton-Cartan background [19].

We conclude this section with the calculation of the Poisson brackets between constraints $\mathcal{H}_{\sigma}, \mathcal{H}_{\tau}$ in order to show that the Poisson algebra is closed. It is convenient to introduce their smeared forms

$$
\mathbf{T}_{\tau}(N)=\int d \sigma N \mathcal{H}_{\tau}, \quad \mathbf{T}_{\sigma}\left(N^{\sigma}\right)=\int d \sigma N^{\sigma} \mathcal{H}_{\sigma}
$$

First of all we have

$$
\left\{\mathbf{T}_{\sigma}\left(N^{\sigma}\right), \mathbf{T}_{\sigma}\left(M^{\sigma}\right)\right\}=\int d \sigma\left(N^{\sigma} \partial_{\sigma} M^{\sigma}-\partial_{\sigma} N^{\sigma} M^{\sigma}\right) p_{M} \partial_{\sigma} x^{M}=\mathbf{T}_{\sigma}\left(N^{\sigma} \partial_{\sigma} M^{\sigma}-\partial_{\sigma} N^{\sigma} M^{\sigma}\right)
$$


that shows that this Poisson bracket vanishes on the constraint surface $\mathcal{H}_{\sigma} \approx 0$. Further we have

$$
\left\{\mathbf{T}_{\sigma}\left(N^{\sigma}\right), \mathcal{H}_{\tau}\right\}=-2 \partial_{\sigma} N^{\sigma} \mathcal{H}_{\tau}-N^{\sigma} \partial_{\sigma} \mathcal{H}_{\tau}
$$

that again vanishes on the constraint surface $\mathcal{H}_{\tau} \approx 0$. These results show that $\mathcal{H}_{\sigma} \approx 0$ is the first class constraint. Finally we calculate Poisson bracket between smeared forms of the constraints $\mathcal{H}_{\tau}$

$$
\begin{aligned}
\left\{\mathbf{T}_{\tau}(N), \mathbf{T}_{\tau}(M)\right\} & =4 \int d \sigma\left(\partial_{\sigma} M N-\partial_{\sigma} N M\right) p_{M}\left(h^{M N} h_{N K}+\tau_{A}^{M} \tau_{K}^{A}\right) \partial_{\sigma} x^{K} \\
& =4 \mathbf{T}_{\sigma}\left(N \partial_{\sigma} M-M \partial_{\sigma} N\right)
\end{aligned}
$$

that again vanishes on the constraint surface $\mathcal{H}_{\sigma} \approx 0$. In summary we find that the Poisson brackets of the primary constraints vanish on the constraint surfaces $\mathcal{H}_{\sigma} \approx 0, \mathcal{H}_{\tau} \approx 0$ and hence they are the first class constraints.

\section{Uniform light-cone gauge}

In this section we study gauge fixed form of the theory whose Hamiltonian was found in previous section. Our goal is to impose uniform light-cone gauge. To do this we follow notation that was used recently in [33]. We firstly select two coordinates $x^{0}=t, x^{9}=\phi$ while $x^{\mu}, \mu, \nu=1,2, \ldots, 8$ label transverse directions. Two abelian isometries are realized by shift in $t$ and $\phi$. As a consequence energy and momentum along these directions are conserved. We further presume that the range of world-sheet coordinate $\sigma$ is $\sigma \in(-r, r)$ where $r$ will be fixed by generalized light-cone gauge. Then the conserved energy and momentum along $\phi$ are given by following formulas

$$
E=\int_{-r}^{r} d \sigma p_{t}, \quad J=\int_{-r}^{r} d \sigma p_{\phi} .
$$

In order to impose uniform-light cone gauge let us introduce light-cone coordinates and momenta

$$
\begin{array}{ll}
x^{-}=\phi-t, & x^{+}=\frac{1}{2}(\phi+t)+\alpha x^{-}, \\
p_{+}=p_{\phi}+p_{t}, & p_{-}=\frac{1}{2}\left(p_{\phi}-p_{t}\right)-\alpha p_{+},
\end{array}
$$

with inverse relations

$$
\begin{aligned}
\phi & =x^{+}+x^{-}\left(\frac{1}{2}-\alpha\right), & t & =x^{+}-x^{-}\left(\frac{1}{2}+\alpha\right) \\
p_{t} & =p_{+}\left(\frac{1}{2}-\alpha\right)-p_{-}, & p_{\phi} & =p_{-}+p_{+}\left(\frac{1}{2}+\alpha\right) .
\end{aligned}
$$

In principle we can insert these formulas into Hamiltonian constraint derived in previous section however final expression is very complicated in the full generality. For that reason we restrict to more tractable examples. Let us start with the case of non-zero components 
of metric $h_{t t}, h_{\phi \phi}$ where all off-diagonal components are zero. In other words the metric $h_{M N}$ has block diagonal form

$$
h_{M N}=\left(\begin{array}{ccc}
h_{t t} & 0 & 0 \\
0 & h_{\phi \phi} & 0 \\
0 & 0 & h_{\mu \nu}
\end{array}\right) .
$$

Clearly the first diagonal block has inverse $\operatorname{diag}\left(h_{t t}^{-1}, h_{\phi \phi}^{-1}\right)$ and consequently we find that $\tau_{t}^{A}=\tau_{\phi}^{A}=0$. Let us further presume that $m_{\mu \phi}=m_{\mu t}=0$ together with $m_{t \phi}=0$. Then the Hamiltonian constraint simplifies considerably

$$
\begin{aligned}
\mathcal{H}_{\tau}= & \mathcal{H}^{\perp}+p_{+} h^{++} p_{+}+2 p_{+} h^{+-} p_{-}+p_{-} h^{--} p_{-}+ \\
& +T^{2} \partial_{1} x^{+} h_{++} \partial_{1} x^{+}+2 T^{2} \partial_{1} x^{+} h_{+-} \partial_{1} x^{-}+T^{2} \partial_{1} x^{-} h_{--} \partial_{1} x^{-}
\end{aligned}
$$

where we defined

$$
\begin{array}{lll}
h^{++}=\left(\frac{1}{2}-\alpha\right)^{2} h^{t t}+\left(\frac{1}{2}+\alpha\right)^{2} h^{\phi \phi}, & h^{--}=h^{t t}+h^{\phi \phi}, \\
h^{+-}=\left(\frac{1}{2}+\alpha\right) h^{\phi \phi}-\left(\frac{1}{2}-\alpha\right) h^{t t}, & h_{--}=\left(\frac{1}{2}-\alpha\right)^{2} h_{\phi \phi}+\left(\frac{1}{2}+\alpha\right)^{2} h_{t t}, \\
h_{++}=h_{t t}+h_{\phi \phi}, & h_{+-}=\left(\frac{1}{2}-\alpha\right) h_{\phi \phi}-\left(\frac{1}{2}+\alpha\right) h_{t t},
\end{array}
$$

and where

$\mathcal{H}^{\perp}=-2 T \tilde{\Pi}_{\mu} \tau_{A}^{\mu} \eta^{A B} \epsilon_{B D} \tau_{\mu}{ }^{D} \partial_{1} x^{\mu}+\tilde{\Pi}_{\mu} h^{\mu \nu} \tilde{\Pi}_{\nu}+T^{2} \partial_{\sigma} x^{\mu} h_{\mu \nu} \partial_{\sigma} x^{\nu}, \quad \tilde{\Pi}_{\mu}=p_{\mu}+T m_{\mu \nu} \partial_{1} x^{\nu}$.

Now we are ready to impose uniform light-cone gauge by introducing following gauge fixed functions [33]

$$
\mathcal{G}^{+} \equiv x^{+}-\left(\tau+a \frac{\pi}{r} m R_{\phi} \sigma\right), \quad \mathcal{G}^{-}=p_{-}-1, \quad a=\frac{1}{2}+\alpha,
$$

where $m$ is integer winding number that represents the number of times the string winds around the circle parametrised by $\phi$. In fact, we implicitly presume that $\phi$ is angular variable $0 \leq \phi \leq 2 \pi R_{\phi}$ and hence

$$
\phi(r)-\phi(-r)=2 \pi m R_{\phi}
$$

Further, if we integrate $p_{-}=1$ over the whole string we can relate $r$ to the total momentum $P_{-}$as

$$
r=\frac{P_{-}}{2} .
$$

Clearly $\mathcal{G}^{+}, \mathcal{G}^{-}$have non-zero Poisson brackets with $\mathcal{H}_{\tau}, \mathcal{H}_{\sigma}$ and hence form set of second class constraints that vanish strongly. As a result constraints $\mathcal{H}_{\tau}=0, \mathcal{H}_{\sigma}=0$ can be explicitly solved. We firstly solve $\mathcal{H}_{\sigma}=0$ for $\partial_{1} x^{-}$and we get

$$
\partial_{\sigma} x^{-}=-p_{\mu} \partial_{\sigma} x^{\mu}-a \frac{\pi}{r} m R_{\phi} p_{+} .
$$


As we argued above $\mathcal{H}_{\tau}, \mathcal{H}_{\sigma}$ vanish strongly and hence gauge fixed form of the action is equal to

$$
\begin{aligned}
S & =\int_{-r}^{r} d \tau d \sigma\left(p_{\mu} \partial_{\tau} x^{\mu}+p_{+} \partial_{\tau} x^{+}+p_{-} \partial_{\tau} x^{-}\right)= \\
& =\int_{-r}^{r} d \tau d \sigma\left(p_{\mu} \partial_{\tau} x^{\mu}+p_{+}\right) .
\end{aligned}
$$

In other words we can identify Hamiltonian density on the reduced phase space as $\mathcal{H}_{\text {red }}=$ $-p_{+}$where $p_{+}$is solution of the Hamiltonian constraint. Explicitly, it is solution of the equation

$$
\begin{aligned}
0= & \mathcal{H}^{\perp}+p_{+} h^{++} p_{+}+2 p_{+} h^{+-}+h^{--}+ \\
& +T_{1}^{2}\left(\frac{a \pi}{r} m R_{\phi}\right)^{2} h_{++}-2 T^{2}\left(\frac{a \pi}{r} m R_{\phi}\right)\left(p_{\mu} \partial_{\sigma} x^{\mu}+a \frac{\pi}{r} m R_{\phi} p_{+}\right) \\
& +T^{2}\left(p_{\mu} \partial_{\sigma} x^{\mu}-a \frac{\pi}{r} m R_{\phi} p_{+}\right) h_{--}\left(p_{\nu} \partial_{\sigma} x^{\nu}+a \frac{\pi}{r} m R_{\phi} p_{+}\right) .
\end{aligned}
$$

This is quadratic equation for $p_{+}$that can be solved at least in principle. For simplicity let us consider the case of the string with zero winding so that $m=0$ and we obtain the result

$$
p_{+}=\frac{-h^{+-}-\sqrt{\left(h^{+-}\right)^{2}-h^{++}\left(\mathcal{H}^{\perp}+h^{--}+T^{2}\left(p_{\mu} \partial_{\sigma} x^{\mu}\right)^{2} h_{--}\right)}}{h^{++}} .
$$

However the form of this expression suggests that this gauge is not well defied due to the presence of - sign in front of $h^{++}$under square root where $h^{++}$is positive by definition. On the other hand it is more natural to impose light cone gauge when we presume non-zero $\tau_{t}{ }^{A}, \tau_{\phi}{ }^{A}$ together with $h_{t t}=h_{t \phi}=h_{\phi \phi}=0$. In other words $h_{M N}$ has non-zero components $h_{\mu \nu}$. We will also presume that the matrix $h_{\mu \nu}$ is non-singular and consequently we have $\tau_{\mu}{ }^{A}=0$. Then the Hamiltonian constraint has the form

$$
\begin{aligned}
\mathcal{H}_{\tau}= & -2 T\left(p_{+}\left(\frac{1}{2}-\alpha\right)-p_{-}+m_{t \phi}\left(\partial_{\sigma} x^{+}+\partial_{\sigma} x^{-}\left(\frac{1}{2}-\alpha\right)\right)\right) \tau_{A}^{t} \eta^{A B} \epsilon_{B D} \times \\
& \times\left(\tau_{t}^{D}\left(\partial_{1} x^{+}-\partial_{1} x^{-}\left(\frac{1}{2}+\alpha\right)\right)+\tau_{\phi}^{D}\left(\partial_{1} x^{+}+\partial_{1} x^{-}\left(\frac{1}{2}-\alpha\right)\right)+\right. \\
& -2 T\left(p_{-}+p_{+}\left(\frac{1}{2}+\alpha\right)+T m_{\phi t}\left(\partial_{1} x^{+}-\partial_{1} x^{-}\left(\frac{1}{2}+\alpha\right)\right) \tau_{A}^{\phi} \eta^{A B} \epsilon_{B D} \times\right. \\
& \times\left(\tau_{t}^{D}\left(\partial_{1} x^{+}-\partial_{1} x^{-}\left(\frac{1}{2}+\alpha\right)\right)+\tau_{\phi}^{D}\left(\partial_{1} x^{+}+\partial_{1} x^{-}\left(\frac{1}{2}-\alpha\right)\right)\right)+ \\
& +\tilde{\Pi}_{\mu} h^{\mu \nu} \tilde{\Pi}_{\nu}++T^{2} \partial_{\sigma} x^{\mu} h_{\mu \nu} \partial_{\sigma} x^{\nu}
\end{aligned}
$$

Now we are ready to fix the gauge. For simplicity we will presume string with zero winding so that gauge fixing functions has the form

$$
\mathcal{G}^{+} \equiv x^{+}-\tau, \quad \mathcal{G}^{-}=p_{-}-1
$$


Then from $\mathcal{H}_{\sigma}=0$ we again get $\partial_{\sigma} x^{-}=-p_{\mu} \partial_{\sigma} x^{\mu}$ and hence Hamiltonian constraint has the form

$$
\begin{aligned}
\mathcal{H}_{\tau}= & -2 T\left(p_{+}\left(\frac{1}{2}-\alpha\right)-1+m_{t \phi}\left(1-p_{\mu} \partial_{\sigma} x^{\mu}\left(\frac{1}{2}-\alpha\right)\right)\right) \tau^{t}{ }_{A} \eta^{A B} \epsilon_{B D} \times \\
& \times\left(\tau_{1}{ }^{D} \partial_{\sigma} x^{\mu} p_{\mu}\left(\frac{1}{2}+\alpha\right)-\tau_{\phi}{ }^{D} p_{\mu} \partial_{\sigma} x^{\mu}\left(\frac{1}{2}-\alpha\right)\right) \\
& -2 T\left(1+p_{+}\left(\frac{1}{2}+\alpha\right)+T m_{\phi t} p_{\mu} \partial_{\sigma} x^{\mu}\left(\frac{1}{2}+\alpha\right)\right) \tau^{\phi}{ }_{A} \eta^{A B} \epsilon_{B D} \times \\
& \times\left(\tau_{1}{ }^{D} \partial_{\sigma} x^{\mu} p_{\mu}\left(\frac{1}{2}+\alpha\right)-\tau_{\phi}{ }^{D} p_{\mu} \partial_{\sigma} x^{\mu}\left(\frac{1}{2}-\alpha\right)\right)+\mathcal{H}^{\perp}=0
\end{aligned}
$$

from which we can express $p_{+}$as

$$
\begin{aligned}
p_{+}= & -\frac{1}{\left(p_{\mu} \partial_{\sigma} x^{\mu}\right)\left(\left(\frac{1}{2}-\alpha\right) \tau_{A}^{t}+\left(\frac{1}{2}+\alpha\right) \tau_{A}^{\phi}\right) \eta^{A B} \epsilon_{B D}\left(\tau_{t}{ }^{D}\left(\frac{1}{2}+\alpha\right)-\tau_{\phi}{ }^{D}\left(\frac{1}{2}-\alpha\right)\right)} \times \\
& \times\left(\left[\left(-1+m_{t \phi}\left(1-p_{\mu} \partial_{\sigma} x^{\mu}\left(\frac{1}{2}-\alpha\right)\right)\right) \tau^{t}{ }_{A}+\left(1+T m_{\phi t} p_{\mu} \partial_{\sigma} x^{\mu}\left(\frac{1}{2}+\alpha\right)\right) \tau^{\phi}{ }_{A}\right] \eta^{A B} \epsilon_{B D}\right. \\
& \left.\times\left(\tau_{1}{ }^{D} \partial_{\sigma} x^{\mu} p_{\mu}\left(\frac{1}{2}+\alpha\right)-\tau_{\phi}{ }^{D} p_{\mu} \partial_{\sigma} x^{\mu}\left(\frac{1}{2}-\alpha\right)\right)+\mathcal{H}^{\perp}\right) .
\end{aligned}
$$

As we argued above $p_{+}=-\mathcal{H}_{\text {red }}$ and hence we have derived Hamiltonian density on the reduced phase space. We see that it has non-relativistic nature since it is quadratic in momenta. This Hamiltonian density could be starting point for further investigation of non-relativistic limit of some string theory backgrounds.

\section{Acknowledgments}

This work is supported by the grant "Integrable Deformations" (GA20-04800S) from the Czech Science Foundation (GACR).

Open Access. This article is distributed under the terms of the Creative Commons Attribution License (CC-BY 4.0), which permits any use, distribution and reproduction in any medium, provided the original author(s) and source are credited.

\section{References}

[1] J. Gomis and H. Ooguri, Nonrelativistic closed string theory, J. Math. Phys. 42 (2001) 3127 [hep-th/0009181] [INSPIRE].

[2] U.H. Danielsson, A. Guijosa and M. Kruczenski, IIA/B, wound and wrapped, JHEP 10 (2000) 020 [hep-th/0009182] [INSPIRE].

[3] R. Andringa, E. Bergshoeff, J. Gomis and M. de Roo, 'Stringy' Newton-Cartan gravity, Class. Quant. Grav. 29 (2012) 235020 [arXiv:1206.5176] [INSPIRE].

[4] T. Harmark, J. Hartong and N.A. Obers, Nonrelativistic strings and limits of the AdS/CFT correspondence, Phys. Rev. D 96 (2017) 086019 [arXiv: 1705.03535] [INSPIRE].

[5] E.A. Bergshoeff, J. Lahnsteiner, L. Romano, J. Rosseel and C. Simsek, Non-relativistic ten-dimensional minimal supergravity, arXiv:2107.14636 [INSPIRE]. 
[6] J. Hartong and E. Have, On the non-relativistic expansion of closed bosonic strings, arXiv: 2107.00023 [INSPIRE].

[7] C.D.A. Blair, D. Gallegos and N. Zinnato, A non-relativistic limit of M-theory and 11-dimensional membrane Newton-Cartan geometry, JHEP 21 (2021) 015 [arXiv:2104.07579] [INSPIRE].

[8] E.A. Bergshoeff, J. Lahnsteiner, L. Romano, J. Rosseel and C. Şimşek, A non-relativistic limit of NS-NS gravity, JHEP 06 (2021) 021 [arXiv: 2102.06974] [INSPIRE].

[9] J. Gomis, Z. Yan and M. Yu, T-duality in nonrelativistic open string theory, JHEP 02 (2021) 087 [arXiv: 2008. 05493] [INSPIRE].

[10] J. Kluson, Stable and unstable Dp-branes in p-brane Newton-Cartan background, J. Phys. A 54 (2021) 215401 [arXiv:2003.14037] [INSPIRE].

[11] Z. Yan and M. Yu, Background field method for nonlinear sigma models in nonrelativistic string theory, JHEP 03 (2020) 181 [arXiv: 1912.03181] [INSPIRE].

[12] E.A. Bergshoeff, J. Gomis, J. Rosseel, C. Şimşek and Z. Yan, String theory and string Newton-Cartan geometry, J. Phys. A 53 (2020) 014001 [arXiv:1907.10668] [InSPIRE].

[13] T. Harmark, J. Hartong, L. Menculini, N.A. Obers and G. Oling, Relating non-relativistic string theories, JHEP 11 (2019) 071 [arXiv:1907.01663] [INSPIRE].

[14] A.D. Gallegos, U. Gürsoy and N. Zinnato, Torsional Newton Cartan gravity from non-relativistic strings, JHEP 09 (2020) 172 [arXiv:1906.01607] [INSPIRE].

[15] J. Gomis, J. Oh and Z. Yan, Nonrelativistic string theory in background fields, JHEP 10 (2019) 101 [arXiv: 1905.07315] [inSPIRE].

[16] J. Klusoň and P. Novosad, Non-relativistic M2-brane, JHEP 06 (2019) 072 [arXiv: 1903.12450] [INSPIRE].

[17] J. Klusoň, Note about T-duality of non-relativistic string, JHEP 08 (2019) 074 [arXiv: 1811.12658] [INSPIRE].

[18] E.A. Bergshoeff, K.T. Grosvenor, C. Simsek and Z. Yan, An action for extended string Newton-Cartan gravity, JHEP 01 (2019) 178 [arXiv: 1810.09387] [INSPIRE].

[19] J. Klusoň, Nonrelativistic string theory sigma model and its canonical formulation, Eur. Phys. J. C 79 (2019) 108 [arXiv:1809.10411] [InSPIRE].

[20] E. Bergshoeff, J. Gomis and Z. Yan, Nonrelativistic string theory and T-duality, JHEP 11 (2018) 133 [arXiv: 1806.06071] [inSPIRE].

[21] J. Kluson, Note about null dimensional reduction of M5-brane, arXiv:2105.13773 [INSPIRE].

[22] J. Kluson, Null dimensional reduction of M2-brane, arXiv:2103.00566 [INSPIRE].

[23] A. Fontanella, J.M. Nieto García and A. Torrielli, Light-cone gauge in non-relativistic $A d S_{5} \times S^{5}$ string theory, arXiv:2102.00008 [INSPIRE].

[24] J. Kluson, Canonical analysis of non-relativistic string with non-relativistic world-sheet, arXiv:2101.03758 [INSPIRE].

[25] T. Harmark, J. Hartong, N.A. Obers and G. Oling, Spin matrix theory string backgrounds and Penrose limits of AdS/CFT, JHEP 03 (2021) 129 [arXiv: 2011.02539] [inSPIRE].

[26] J. Klusoň, Unstable D-brane in torsional Newton-Cartan background, JHEP 09 (2020) 191 [arXiv:2001.11543] [INSPIRE]. 
[27] D. Hansen, J. Hartong and N.A. Obers, Non-relativistic gravity and its coupling to matter, JHEP 06 (2020) 145 [arXiv:2001.10277] [INSPIRE].

[28] J. Klusoň, T-duality of non-relativistic string in torsional Newton-Cartan background, JHEP 05 (2020) 024 [arXiv: 1909.13508] [INSPIRE].

[29] J. Klusoň, Non-relativistic D-brane from T-duality along null direction, JHEP 10 (2019) 153 [arXiv: 1907.05662] [INSPIRE].

[30] D. Hansen, J. Hartong and N.A. Obers, Action principle for Newtonian gravity, Phys. Rev. Lett. 122 (2019) 061106 [arXiv: 1807.04765] [INSPIRE].

[31] J. Klusoň, Remark about non-relativistic string in Newton-Cartan background and null reduction, JHEP 05 (2018) 041 [arXiv:1803.07336] [INSPIRE].

[32] L. Bidussi, T. Harmark, J. Hartong, N.A. Obers and G. Oling, Torsional string Newton-Cartan geometry for non-relativistic strings, arXiv:2107.00642 [INSPIRE].

[33] S. Frolov, $T \bar{T}$ deformation and the light-cone gauge, Proc. Steklov Inst. Math. 309 (2020) 107 [arXiv: 1905. 07946] [INSPIRE].

[34] G. Arutyunov and S. Frolov, Foundations of the $A d S_{5} \times S^{5}$ superstring. Part I, J. Phys. A 42 (2009) 254003 [arXiv:0901.4937] [INSPIRE].

[35] G. Arutyunov, S. Frolov and M. Zamaklar, Finite-size effects from giant magnons, Nucl. Phys. B $\mathbf{7 7 8}$ (2007) 1 [hep-th/0606126] [INSPIRE].

[36] S. Frolov, J. Plefka and M. Zamaklar, The AdS $S_{5} \times S^{5}$ superstring in light-cone gauge and its Bethe equations, J. Phys. A 39 (2006) 13037 [hep-th/0603008] [InSPIRE].

[37] G. Arutyunov and S. Frolov, Uniform light-cone gauge for strings in $A d S_{5} \times S^{5}$ : solving SU(1|1) sector, JHEP 01 (2006) 055 [hep-th/0510208] [INSPIRE]. 